\title{
THE STUDY OF HYDRODYNAMIC PROCESSES AT THE GAS FLOW FILTRATION THROUGH THE CANDIED FRUITS LAYER
}

\author{
Volodymyr Atamanyuk \\ Department of Chemical Engineering \\ National University "Lviv Polytechnic» \\ 12 Bandera str., Lviv, Ukraine, 79013 \\ atamanyuk@ukr.net \\ Iryna Huzova \\ Department of Chemical Engineering \\ National University "Lviv Polytechnic» \\ 12 Bandera str., Lviv, Ukraine, 79013 \\ iryna.o.huzova@lpnu.ua \\ Zoriana Gnativ \\ Department of Chemical Engineering \\ National University "Lviv Polytechnic» \\ 12 Bandera str., Lviv, Ukraine, 79013 \\ zorchyk@gmail.com \\ Boris Mykychak \\ Lviv College of Meat and Dairy Industry (LCMDI NUFT) \\ 30/32 Bortnyansky str., Lviv, Ukraine, 79000 \\ borys.mykychak@gmail.com
}

\begin{abstract}
The experimental studies of hydrodynamics of the gas flow filtration through the candied fruits layer, formed by the different methods, were carried out. The particles of studied candied fruits were cylindrical, equally sized, filled the whole container for drying. The main characteristics of the layers were experimentally determined: porosity, equivalent diameter, specific surface. The results of experiments prove the expedience of the candied fruits layer formation of the cylindrical particles with correct form by the method of placing candied fruits vertically "with overlap of channels". Such method allows provide the insignificant hydraulic resistance of the layer and rather high speed of the gas flow filtration through this layer. It will result in the increase of heat transfer and mass output coefficients, so to the intensification of filtration drying. Such formation method also allows reduce the volume of drying equipment, shorten the time of drying and decrease the energy consumption of process.
\end{abstract}

Keywords: candied fruit, layer formation, hydraulic resistance, speed, filtration, equivalent diameter.

\section{Introduction}

The candied fruits production is considered as a priority direction of food industry [1]. The limiting and the most power-consuming stage of the candied fruits production is the drying process. The drying time of candied fruits depends on both drying method and size and form of particles [2].

The particles of vegetables and fruits for candied fruits production are cut in the different form. The form of particle depends, in first turn, on the nature of raw material. The most spread form of particles for candied fruits production is cubs, cylinder, pieces of rectangular or incorrect form [3-5].

The most widespread methods of candied fruits drying are convective, sublimation and combined ones [2]. At that the candied fruits particles are placed on the belts or sieves of drying equipment chaotically [6,7].

The method of disperse materials drying by filtration through the chaotically formed layer is considered in the work [8]. The other methods of the layer formation at any drying method are presented insufficiently widely in scientific literature. The gas flow filtration through the chaotically 
formed layer is realized unevenly on the whole height of the layer. It is a cause of uneven drying and as the result, the increase of energy consumption and worsening of the ready production quality. That is just a cause of the interest to the studies of the influence of the ordered formation of candied fruits layer on the working surface of drying equipment on hydrodynamic processes. Such studies are not widely presented in scientific literature.

The speed of gas flow filtration through the layer of candied fruits particles determines intensity and economic expedience of the process of filtration drying of wet material. There is a close connection between the drying intensity and hydrodynamics of the process. The movement character of gas flow, filtered through the layer, determines the movement regimes and heat transfer and mass output coefficients. The increase of the speed of gas medium movement relative to the solid surface leads to intensification of the drying. In chaotically formed layer it is impossible to attain the equal speed on its whole height.

In practice it is possible to realize the increase of gas flow speed in the layer of material by the decrease of equivalent diameter between particles and by its equal formation on the whole height of the layer.

It can be attained by the formation of the ordered layer of particles with equal forms and size.

From the other side, the significant speeds of gas flow in the formed layer will result in the increase of its hydraulic resistance. That is why the choice of the optimal method of layer formation is a topical problem.

Based on it, the aim of the work is in following: the choice of the optimal method of candied fruits layer formation that allows decrease the energy consumption and raise the quality of ready production.

\section{Materials and Methods}

The material of research was the pumpkin particles of the different sizes, cylindrical form, with height $20 \mathrm{~mm}$ and diameter $10 \mathrm{~mm}$ (Fig. 1).

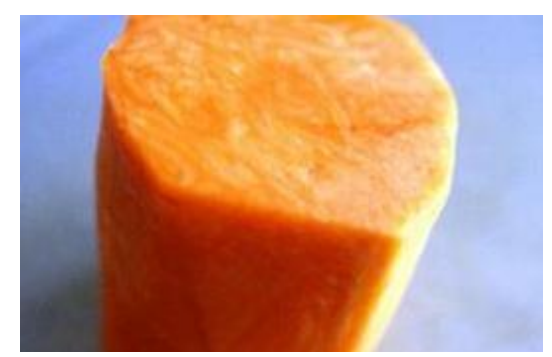

Fig. 1. Cylindrical form of the studied sample

The surface of cylinder is smooth, external fissures are absent. Cylinder has insignificant form factor and allows reduce the parietal effect in apparatus. The pumpkin sort "Stofuntovka Vassma", planted in the Western regions of Ukraine.

Experimental studies were carried out in the container, which scheme is presented on the Fig. 2.

The general container of cylindrical form for the study of hydrodynamics consisted of four containers with height $30 \mathrm{~mm}$, each of them corresponds to the scheme, presented on the Fig. 2. Containers and perforated barriers are made of fluoroplastic 4D [9]. The pressure drop in material layer was fixed by differential manometer OKCI 1D. The depression of instrument is 1-1000 Pa; the instrument sensitivity is $1 \mathrm{~Pa}$. The country-producer is China; the firm-producer is CEM R \& $\mathrm{D}$. The gas flow consumption was fixed by rotameter of the basic model RM-II, model RM-02-0,016HZUZ. Country-producer is Russia (Arzamas instrument-making plant).

The temperature was fixed by potentiometer CR-7260. The country-producer is China. The instrument accuracy is $\pm 0,4{ }^{\circ} \mathrm{C}$. Experiments were carried out at the fixed temperature $50{ }^{\circ} \mathrm{C}$. The studies of candied fruits drying in the soft regime were taken as the base $[10,11]$. 


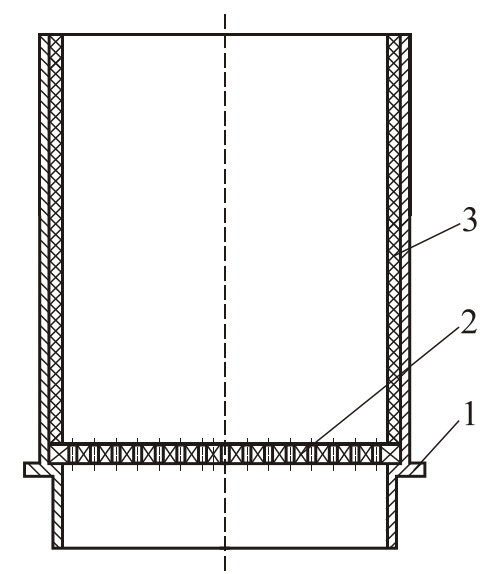

Fig. 2. Scheme of container for the loading of candied fruits particles: 1 - body of container,

2 - perforated barrier, 3 - heat-insulated inset

Preparation of the raw material was realized as following: pumpkin was washed peeled of rind and film, cut in whole pieces with height $\geq 20 \mathrm{~mm}$. With the help of matrix the cylindrical particles with equal sizes $(20 \times 10)$ were formed. The layers were formed of the received particles in containers (Fig. 2) by the four different methods (Fig. 3).

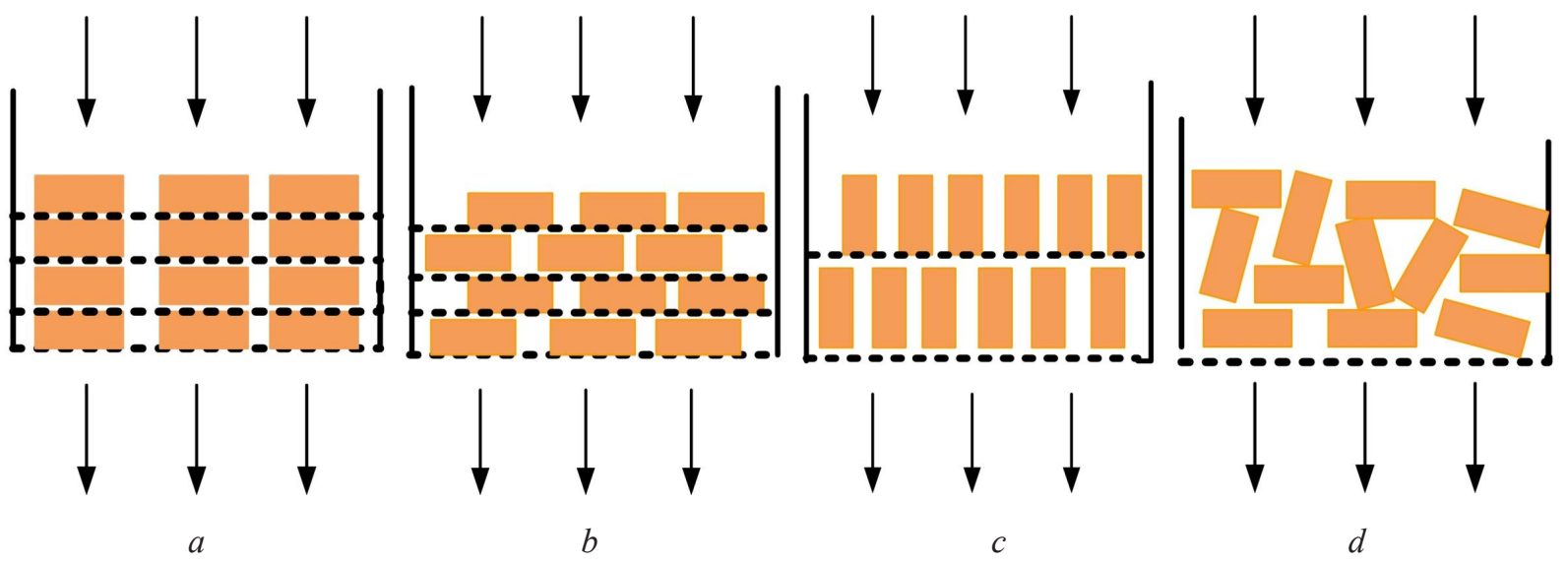

Fig. 3. Methods of formation of the candied fruits layer: $a$ - I method of candied fruits placement "face to face"; $b$ - II method of candied fruits placement horizontally "with overlap of channels"; $c$ - III method of candied fruits placement vertically "with overlap of channels"; $d$ - IV method of candied fruits placement "chaotically"

The experiment was carried out at the different heights of the layer (20-120 mm). The fixed height at each method was formed of the different number of particles. The formed layers filled the whole container. Only at such placement (Fig. 3) it is possible to attain the equivalent diameter between particles on the whole height of the layer. The equivalent diameter was calculated according to the well-known formula [12]:

$$
\mathrm{d}_{\mathrm{e}}=\frac{4 \cdot \varepsilon}{\mathrm{a}}
$$

where $\varepsilon$ - porosity of the layer, $\mathrm{m}^{3} / \mathrm{m}^{3} ; \mathrm{a}$ - specific surface of the layer, $\mathrm{m}^{2} / \mathrm{m}^{3}$.

Porosity and specific surface of the layer at its formation of particles with the equal size and form are calculated according to the elementary geometric dependences. 


\section{1. Experimental procedures}

Experimental studies of the gas flow filtration through the formed layer of candied fruits were carried out on the setting, presented on the Fig. 4.

The ventilator 8 and heater 7 were switched on. Container 1 was set on the receiver 2. Shutoff valve 4 was open. Vacuum pump 6 was switched on and the different consumption of the gas flow through the layer was put by the regulating valve 5. Gas flow was filtered in direction "layer of pumpkin particles-perforated barrier" under the effect of pressure drop. The value of consumption was determined by the figures of rotameter 3, and pressure losses - by the figures of differential manometer 12 . The temperature was fixed by potentiometer 11, using thermocouple 9.

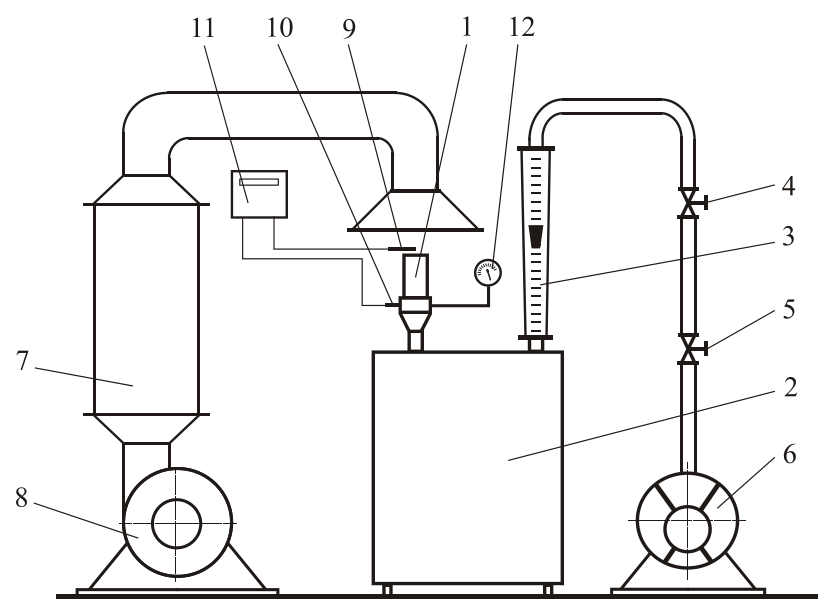

Fig. 4. Scheme of experimental setting: 1 - container, 2 - receiver, 3 - rotameter,

4,5 - shutoff and regulating valves, 6 - liquid ring vacuum pump, 7 - heater, 8 - ventilator, 9,10 - thermocouples, 11 - instrument for temperature measurement, 12 -differential manometer

Each experiment was carried out no less than 3 times. The fresh particles were selected for each experiment. The error of values of the different experiments did not exceed $5 \%$.

\section{Results}

The experimentally determined values of porosity, specific surface and equivalent diameter of the layer for I-IV methods are given in the Table $\mathbf{1 .}$

Table 1

Characteristics of the candied fruits layer, formed by the different methods

\begin{tabular}{ccccc}
\hline Layer characteristics & I method & II method & III method & IV method \\
\hline$\varepsilon, \mathrm{m}^{3} / \mathrm{m}^{3}$ & 0.23 & 0.23 & 0.23 & 0.1 \\
$\mathrm{a}, \mathrm{m}^{2} / \mathrm{m}^{3}$ & 178,5 & 244 & 277 & 172 \\
$\mathrm{~d}_{\mathrm{e}}, \mathrm{m}$ & 0,005 & 0,004 & 0,003 & 0,002
\end{tabular}

From the Table 1 we can see that the third method of the layer formation has the maximal specific surface and least equivalent diameter comparing with two first methods. So, we can presuppose that the contact between the gas flow and candied fruits particles will be maximal and the gas speed in the layer will be rather high.

The results of the dependency of the pressure losses in the layer of candied fruits, placed according to the four methods, on the fictitious speed of the gas flow are presented on the Fig. 5 .

As we can see on the Fig. 5 (curves 1, 4), the layers with the least surface of phases contact have the least resistance (Table 1). The layer, formed by the III method (curve 3 ) has the less resistance than the layer, formed by the II method (curve 2). 


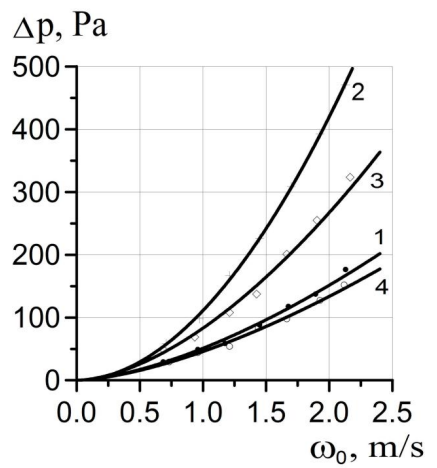

Fig. 5. The dependency of the pressure losses in the layer of candied fruits, placed according to the four methods, on the fictitious speed of the gas flow ( $\mathrm{H}=0,04 \mathrm{~m}$ (56 particles)): 1 - I method;

2 - II method; 3 - III method; 4 - IV method

\section{Conclusions}

The offered experimental studies indicate the necessity of drying candied fruits in the package of systemically formed layers. Such drying method can be introduced in production after enrichment of experimental data.

The experiments prove the expedience of the candied fruits layer formation of the cylindrical particles of correct form by the method of placing candied fruits vertically "with overlap of channels". Such method allows provide the insignificant hydraulic resistance of the layer and rather high speed of the gas flow filtration through this layer. It would result in the increase of heat transfer and mass output coefficients, so to the intensification of filtration drying. Such formation method also allows reduce the volume of drying equipment, shorten the time of drying.

On the base of experimental data the necessity of further calculation, modeling and optimization of the values of hydraulic resistance of the layer and the gas flow speed in it appeared.

\section{References}

[1] Dolmatova, I., Zaitseva, T., Malova, E (2013). Development of technology of candied fruit garden nightshade Sunberry. The collection of scientific papers "Magnitogorsk State Technical University. GI Nosov", 233-236.

[2] Alibas, I. (2006). Characteristics of Chard Leaves during Microwave, Convective, and Combined Microwave-Convective Drying. Drying Technology, 24 (11), 1425-1435. doi: 10.1080/07373930600952776

[3] Zakharenko, V., Nepochatykh, T. (2003). The method of manufacturing candied pumpkin and carrots. Patent 57419A. MPK A23G 3/48. 2002107839; declareted: 03.10.2002; published: 16.06.2003, Bul. 6.

[4] Cherevko, A., Mikhailov, V., Mayak, V. (2005). A process for preparing candied fruits. Patent 6435. MPK A23L 1/06. 20040806738; declareted: 11.08.2004; published: 16.05.2005, Byul. 5.

[5] Malezhik, I., Dubkovetskyy, I., Bandurenko, G., Strelchenko, L. (2015). The method of manufacturing a candied apple. Patent 103371. MPK A23B 7/02. u201506572; declareted: 03.07.2015; published: 10.12.2015, Bul. 23.

[6] Yarovyj, I. I., Katasonov, O. V. (2015). Some problems of experimental modeling process drying plant material in a microwave electromagnetic field. Proceedings ONAFT, 47 (2), 227-231.

[7] Dubkovetskyy, I., Malezhik, I. F., Strelchenko, L. V., Yevchuk, Ya. V. (2015). Investigation of the kinetics of convection - drying termoradiatsiynoho hawthorn. Proceedings ONAFT, 47 (2), 97-104.

[8] Atamanyuk, V. N., Huzova, I. A., Patrij, N. I. (2016). Hydrodynamics sludge drying coffee. Chemical Industry of Ukraine, 2 (133), 12-17

[9] GOST 10007-80. Teflon-4. Specifications (1996). Moscow: Publishing House of Standards, 31.

[10] Nepochatykh, T. (2016). Some theoretical and practical approaches to the production of candied fruits from plant material. Science trajectory, 1 (30), 2.1-2.8.

[11] Abraão, A. S., Lemos, A. M., Vilela, A., Sousa, J. M., Nunes, F. M. (2013). Influence of osmotic dehydration process parameters on the quality of candied pumpkins. Food and Bioproducts Processing, 91 (4), 481-494. doi: 10.1016/j.fbp.2013.04.006

[12] Atamanyuk, V. (2013). Scientific basis filtration drying dispersed materials. Lviv, 340. 